\title{
ON THE LINEAR FRACTIONAL SELF-ATTRACTING DIFFUSION*
}

\author{
LITAN YAN, YU SUN AND YUNSHENG LU
}

\author{
Department of Mathematics, Donghua University,
} 2999 North Renmin Rd. Shanghai 201620, P. R. China.

\begin{abstract}
In this paper, we introduce the linear fractional self-attracting diffusion driven by a fractional Brownian motion with Hurst index $1 / 2<H<1$, which is analogous to the linear self-attracting diffusion. For 1-dimensional process we study its convergence and the corresponding weighted local time. For 2-dimensional process, as a related problem, we show that the renormalized selfintersection local time exists in $L^{2}$ if $\frac{1}{2}<H<\frac{3}{4}$.
\end{abstract}

\section{INTRODUCTION}

In 1991, Durrett and Rogers [7] studied a system that models the shape of a growing polymer. Under some conditions, they established asymptotic behavior of the solution of stochastic differential equation

$$
X_{t}=B_{t}+\int_{0}^{t} \int_{0}^{s} \Phi\left(X_{s}-X_{u}\right) d u d s
$$

where $B$ is a $d$-dimensional standard Brownian motion and $\Phi$ Lipschitz continuous. If $\Phi(x)=\Psi(x) x /\|x\|$ and $\Psi(x) \geq 0, X_{t}$ is a continuous analogue of a process introduced by Diaconis and studied by Pemantle [20]. The path dependent stochastic differential equation can be considered as polymer model. In 1995, Cranston and Le Jan [5] extended the model and introduced self-attracting diffusions, where for $d=1$ two cases of are studied: the linear interaction where $\Phi$ is a linear function and the constant interaction in dimension 1 , where $\Phi(x)=\sigma \operatorname{sign}(x)$ for positive $\sigma$, and in both cases the almost sure convergence of $X_{t}$ is proved. Herrmanna-Roynette [9], Herrmanna-Scheutzowb [10] generalized these results.

On the other hand, the statistical properties of fractional Brownian motion (fBm) are used to construct a path integral representation of the conformations of some polymers (see, for examples, Chakravarti and Sebastian [3], Cherayil and Biswas [4], Sebastian [21]). Thus, as a natural extension to (1.1) one may consider the path

${ }^{*}$ The Project-sponsored by NSFC (10571025) and the Key Project of Chinese Ministry of Education (No.106076).

2000 Mathematics Subject Classification. 60G15, 60J55, 60H05.

Key words and phrases. fractional Brownian motion, self-attracting diffusion, the fractional Itô integrals, local time and self-intersection local time. 
dependent stochastic differential equation of the form

$$
X_{t}^{H}=B_{t}^{H}+\int_{0}^{t} \int_{0}^{s} \Phi\left(X_{s}^{H}-X_{u}^{H}\right) d u d s
$$

where $B^{H}$ is a $d$-dimensional fractional Brownian motion with Hurst index $H \in$ $(0,1)$ and $\Phi$ Lipschitz continuous. Then it is not difficult to show that the above equation admits a unique strong solution. We will call the solution the fractional self-attracting diffusion driven by $\mathrm{fBm}$. In this paper, we consider only a particular case as follows (the linear fractional self-attracting diffusion):

$$
X_{t}^{H}=B_{t}^{H}-a \int_{0}^{t} \int_{0}^{s}\left(X_{s}^{H}-X_{u}^{H}\right) d u d s+\nu t
$$

with $a>0, \nu \in \mathbb{R}^{d}$ and $\frac{1}{2}<H<1$. Our aims are to study the convergence and local times of the processes given by (1.3) with $d=1$. As a related problem, for the two dimensional process we shall show that the renormalized self-intersection local time exists in $L^{2}$ if $\frac{1}{2}<H<\frac{3}{4}$.

The structure of this paper is as follows. In Section 2 we briefly recall $\mathrm{fBm}$ and related the Itô type stochastic integral. In Section 3 we investigate convergence of the linear fractional self-attracting diffusion. We show that the process converges with probability one as $t$ tends to infinity. In Section 4, we define the weighted local time of the process and obtain a Meyer-Tanaka type formula. Finally, in Section 5 for 2-dimensional process we show that its renormalized self-intersection local time exists in $L^{2}$ if $\frac{1}{2}<H<\frac{3}{4}$.

\section{Fractional Brownian motion and the Itô type formula}

In this section, we briefly recall the definition and properties of stochastic integral with respect to $\mathrm{fBm}$. Throughout this paper we assume that $\frac{1}{2}<H<1$ is arbitrary but fixed. Let $(\Omega, \mathcal{F}, \mu)$ be a complete probability space such that a fractional Brownian motion with Hurst index $H$ is well defined. For simplicity we let $C$ stand for a positive constant depending only on the subscripts and its value may be different in different appearance, and this assumption is also adaptable to $c$.

Recall that a centered continuous Gaussian process $B^{H}=\left\{B_{t}^{H}, t \geq 0\right\}$ with the covariance function

$$
E\left[B_{t}^{H} B_{s}^{H}\right]=\frac{1}{2}\left(t^{2 H}+s^{2 H}-|t-s|^{2 H}\right), s, t \geq 0,
$$

is called the $\mathrm{fBm}$ with Hurst index $H$. Here $E$ denotes the expectation with respect to the probability law of $B^{H}$ on $\Omega$. This process was first introduced by Kolmogorov and studied by Mandelbrot and Van Ness [16], where a stochastic integral representation in terms of a standard Brownian motion was established. The definition of stochastic integrals with respect to the $\mathrm{fBm}$ has been investigated by several authors. Here, we refer to Duncan et al [6] and Hu-Øksendal [15] (see also Elliott-Van der Hoek [8], Hu [1], Nualart [17, 18], Øksendel [19]) for the definition and the 
properties of the fractional Itô integral

$$
\int_{0}^{t} u_{s} d B_{s}^{H}
$$

of an adapted process $u$. For $1 / 2<H<1$ we define the function $\phi: \mathbb{R}_{+} \times \mathbb{R}_{+} \rightarrow \mathbb{R}_{+}$ by

$$
\phi(s, t)=H(2 H-1)|s-t|^{2 H-2}, \quad s, t \geq 0 .
$$

Recall that the Malliavin $\phi$-derivative of the function $U: \Omega \rightarrow \mathbb{R}$ defined in [6] as follows:

$$
D_{s}^{\phi} U=\int_{0}^{\infty} \phi(r, s) D_{r} U d r
$$

where $D_{r} U$ is the fractional Malliavin derivative at $r$. Define the space $\mathbb{L}_{\phi}^{1,2}$ to be the set of measurable processes $u$ such that $D_{t}^{\phi} u_{s}$ exists for a.a. $s, t \geq 0$ and

$$
\|u\|_{\mathbb{L}_{\phi}^{1,2}}^{2}:=E\left[\int_{0}^{\infty} \int_{0}^{\infty} D_{s}^{\phi} u_{t} D_{t}^{\phi} u_{s} d s d t+\int_{0}^{\infty} \int_{0}^{\infty} u_{s} u_{t} \phi(s, t) d s d t\right]<\infty .
$$

Thus, the integral $\int_{0}^{\infty} u_{s} d B_{s}^{H}$ can be well defined as an element of $L^{2}(\mu)$ if $u$ satisfies (2.1). For the integral process $\eta_{t}=\int_{0}^{t} u_{s} d B_{s}^{H}$, we have (see, for examples, Duncan et al [6], $\mathrm{Hu}$ [11])

$$
D_{s}^{\phi} \eta_{t}=\int_{0}^{t} D_{s}^{\phi} u_{r} d B_{r}^{H}+\int_{0}^{t} u_{r} \phi(s, r) d r
$$

In particular, if $u$ is deterministic, then $D_{s}^{\phi} \eta_{t}=\int_{0}^{t} u_{r} \phi(s, r) d r$.

Theorem 2.1 (Duncan et al [6], Hu [1]). Let $F \in C^{2}(\mathbb{R})$ having polynomial growth and let the process $X$ be given as follows:

$$
d X_{t}=v_{t} d t+u_{t} d B_{t}^{H}, \quad X_{0}=x \in \mathbb{R},
$$

where $u \in \mathbb{L}_{\phi}^{1,2}$ and measurable process $v$ satisfies $\int_{0}^{t}\left|v_{s}\right| d s<\infty$ a.s. Then we have, for all $t \geq 0$

$$
F\left(X_{t}\right)=F(x)+\int_{0}^{t} \frac{\partial}{\partial x} F\left(s, X_{s}\right) d X_{s}+\int_{0}^{t} \frac{\partial^{2}}{\partial x^{2}} F\left(s, X_{s}\right) u_{s} D_{s}^{\phi} X_{s} d s .
$$

\section{Convergence}

In this section, we consider convergence of the solution of the equation (1.3), the so-call linear fractional self-attracting diffusion. The method used here is essentially due to M. Cranston and Y. Le Jan [5].

Proposition 3.1. The solution to the equation (1.3) can be expressed as

$$
X_{t}^{H}=X_{0}^{H}+\int_{0}^{t} h(t, s) d B_{s}^{H}+\nu \int_{0}^{t} h(t, s) d s
$$

where

$$
h(t, s)= \begin{cases}1-a s e^{\frac{1}{2} a s^{2}} \int_{s}^{t} e^{-\frac{1}{2} a u^{2}} d u, & t \geq s, \\ 0, & t<s\end{cases}
$$

for $s, t \geq 0$. 
This proposition can also be obtained by the same method as Cranston and Le Jan [5]. It follows from the Ito type formula that

$$
\begin{aligned}
F\left(X_{t}^{H}\right)= & F(0)+\int_{0}^{t} F^{\prime}\left(X_{s}^{H}\right) d X_{s}^{H}+\int_{0}^{t} F^{\prime \prime}\left(X_{s}^{H}\right) D_{s}^{\phi} X_{s}^{H} d s \\
= & F(0)+\int_{0}^{t} F^{\prime}\left(X_{s}^{H}\right) d X_{s}^{H} \\
& \quad+2 H(2 H-1) \int_{0}^{t} F^{\prime \prime}\left(X_{s}^{H}\right) d s \int_{0}^{s} h(s, m)(s-m)^{2 H-2} d m
\end{aligned}
$$

for $F \in C^{2}(\mathbb{R})$ having polynomial growth. On the other hand, an elementary calculation yields

$$
h(s)=\lim _{t \uparrow \infty} h(t, s)=1-a s e^{\frac{a}{2} s^{2}} \int_{s}^{\infty} e^{-\frac{a}{2} u^{2}} d u
$$

which is continuous on $[0, \infty)$.

Theorem 3.2. The solution $X_{t}^{H}$ to (1.3) converges in $L^{2}(\mu)$ to the following element as $t \rightarrow \infty$ :

$$
X_{\infty}^{H} \equiv \int_{0}^{\infty} h(s) d B_{s}^{H}+\nu \int_{0}^{\infty} h(s) d s
$$

Proof. Clearly, we have

$$
\left|h\left(t, s_{1}\right)-h\left(s_{1}\right)\right|\left|h\left(t, s_{2}\right)-h\left(s_{2}\right)\right| \leq \frac{1}{t^{2}} s_{1} s_{2} e^{\frac{a}{2}\left(s_{1}^{2}+s_{2}^{2}\right)} e^{-a t^{2}}
$$

for $s_{1}, s_{2} \leq t$ and $\left|\int_{0}^{t}[h(t, s)-h(s)] d s\right| \leq \frac{1}{a t} \rightarrow 0 \quad(t \rightarrow \infty)$. From (2.1) it follows that

$$
E\left|\int_{0}^{t}[h(t, s)-h(s)] d B_{s}^{H}\right|^{2} \leq \frac{2 H}{a t^{2-2 H}} \rightarrow 0
$$

as $t \rightarrow \infty$, which proves

$$
E\left|X_{t}^{H}-X_{\infty}^{H}\right|^{2} \leq 2 E\left|\int_{0}^{t}[h(t, s)-h(s)] d B_{s}^{H}\right|^{2}+2\left|\int_{0}^{t}[h(t, s)-h(s)] d s\right|^{2} \rightarrow 0 .
$$

This completes the proof.

Theorem 3.3. The solution $X_{t}^{H}$ to (1.3) converges to $X_{\infty}^{H}$ almost surely as $t \rightarrow \infty$.

Proof. Without loss of generality, we may assume $\nu=0$. Note that by Proposition 3.1

$$
\begin{aligned}
X_{t}^{H}-X_{\infty}^{H} & =\int_{0}^{t}[h(t, s)-h(s)] d B_{s}^{H}-\int_{t}^{\infty} h(s) d B_{s}^{H} \\
& \equiv Y_{t}^{H}-\int_{t}^{\infty} h(s) d B_{s}^{H}, \quad t \geq 0 .
\end{aligned}
$$

Thus, it is enough to show that $Y_{t}^{H}$ converges to 0 almost surely as $t \rightarrow \infty$. 
For integer numbers $n, k, 0 \leq k<n$ we set $Z_{n, k}^{H}=Y_{n+\frac{k}{n}}^{H}$. Then $Z_{n, k}^{H}$ is Gaussian, and we have

$$
E\left[\left(Z_{n, k}^{H}\right)^{2}\right]=E\left[\left.|| \int_{0}^{n+\frac{k}{n}}[h(t, s)-h(s)] d B_{s}^{H}\right|^{2}\right] \leq \frac{2 H}{a n^{2-2 H}}
$$

by (3.4), and for any $\varepsilon>0$

$$
P\left(\left|Z_{n, k}^{H}\right|>\varepsilon\right) \leq 2 \frac{e^{-\frac{1}{4 H} \varepsilon^{2} a n^{2-2 H}}}{\varepsilon n^{1-H} \sqrt{a \pi}} .
$$

Furthermore, for $s \in(0,1)$ we set $R_{s}^{n, k}=Y_{n+\frac{k+s}{n}}-Y_{n+\frac{k}{n}}$. Then $R_{s}^{n, k}, 0 \leq s \leq 1$ is a Gaussian process and

$$
E\left[\left(R_{s}^{n, k}-R_{s^{\prime}}^{n, k}\right)^{2}\right] \leq \frac{\left|s-s^{\prime}\right|^{2 H}}{n^{2 H}}(1+a)=\frac{1+a}{n^{2 H}} E\left[\left(B_{s}^{H}-B_{s^{\prime}}^{H}\right)^{2}\right]
$$

by (3.4). For any $\varepsilon>0$,

$$
\begin{aligned}
P\left(\sup _{0 \leq s \leq 1}\left|R_{s}^{n, k}\right|>\varepsilon\right) & \leq P\left(\frac{\sqrt{1+a}}{n^{H}} \sup _{0 \leq s \leq 1}\left|B_{s}^{H}\right|>\varepsilon\right) \quad \text { (by Slepian's lemma) } \\
& \leq(1+a) \frac{E\left[\sup _{0 \leq s \leq 1}\left|B_{s}^{H}\right|^{2}\right]}{\varepsilon^{2} n^{2 H}} \\
& \leq C_{H}(1+a) \frac{1}{\varepsilon^{2} n^{2 H}} .
\end{aligned}
$$

Thus, the convergence with probability one follows from the Borel-Cantelli Lemma and

$$
\left\{\sup _{n+\frac{k}{n}<t<n+\frac{k+1}{n}}\left|Y_{t}\right|>\varepsilon\right\} \subseteq\left\{\left|Z_{n, k}^{H}\right|>\varepsilon / 2\right\} \cup\left\{\sup _{0 \leq s \leq 1}\left|R_{s}^{n, k}\right|>\varepsilon / 2\right\}
$$

for all $k, n \geq 0$. This completes the proof of the theorem.

\section{Local time AND Meyer-TANaKa type Formula}

In this section, we consider the linear fractional self-attracting diffusion $X^{H}=$ $\left\{X_{t}^{H}, 0 \leq t \leq T\right\}$ with $\nu=0$. It follows that the process is a centered Gaussian process. We study the usual local time and weighted local time of the process and obtain the Meyer-Tanaka type formula of the weighted local time.

For $T \geq t \geq s \geq 0$ we put

$$
\sigma_{t}^{2}=E\left[\left(X_{t}^{H}\right)^{2}\right], \quad \sigma_{t, s}^{2}=E\left[\left(X_{t}^{H}-X_{s}^{H}\right)^{2}\right] .
$$

Then

and

$$
\sigma_{t}^{2}=\int_{0}^{t} \int_{0}^{t} h(t, u) h(t, v) \phi(u, v) d u d v, \quad 0 \leq t \leq T
$$

$$
\sigma_{t, s}^{2}=\int_{0}^{t} \int_{0}^{t}[h(t, u)-h(s, u)][h(t, v)-h(s, v)] \phi(u, v) .
$$

Noting that for all $t \geq s \geq 0$,

$$
\int_{0}^{t} \int_{0}^{t} \phi(u, v) d u d v=t^{2 H}, \quad e^{-\frac{a}{2}\left(t^{2}-s^{2}\right)} \leq h(t, s) \leq 1,
$$


we get

$$
e^{-\frac{a}{2} t^{2}} t^{2 H} \leq \sigma_{t}^{2}=\int_{0}^{t} \int_{0}^{t} h(t, u) h(t, v) \phi(u, v) d u d v \leq t^{2 H} .
$$

Lemma 4.1. For all $t \geq s \geq 0$ we have

$$
c_{a, H, T}(t-s)^{2 H} \leq \sigma_{t, s}^{2} \leq C_{a, H, T}(t-s)^{2 H},
$$

where $C_{a, H, T}, c_{a, H, T}>0$ are two constants depending on $a, H, T$.

Proof. For all $t \geq s \geq 0$ we have

$$
\begin{aligned}
\sigma_{t, s}^{2}= & \int_{0}^{t} \int_{0}^{t}[h(t, u)-h(s, u)][h(t, v)-h(s, v)] \phi(u, v) d u d v \\
= & \int_{s}^{t} \int_{s}^{t} h(t, u) h(t, v) \phi(u, v) d u d v+ \\
& \quad \int_{s}^{t} \int_{0}^{s} h(t, u)[h(t, v)-h(s, v)] \phi(u, v) d u d v+ \\
& \int_{0}^{s} \int_{s}^{t}[h(t, u)-h(s, u)] h(t, v) \phi(u, v) d u d v+ \\
& \int_{0}^{s} \int_{0}^{s}[h(t, u)-h(s, u)][h(t, v)-h(s, v)] \phi(u, v) d u d v \\
\equiv & A_{[s, t]^{2}}+A_{[s, t] \times[0, s]}+A_{[0, s] \times[s, t]}+A_{[0, s]^{2} .}
\end{aligned}
$$

On the other hand, for all $t \geq s \geq 0$ we have

$$
\begin{aligned}
A_{[s, t] \times[0, s]} & =A_{[0, s] \times[s, t]} \\
& =-\left(\int_{s}^{t} e^{-\frac{a}{2} w^{2}} d w\right) \int_{0}^{s} a u e^{\frac{a}{2} u^{2}} d u \int_{s}^{t} h(t, v) \phi(u, v) d v, \\
A_{[0, s]^{2}}= & 2\left(\int_{s}^{t} e^{-\frac{a}{2} w^{2}} d w\right)^{2} \int_{0}^{s} a^{2} u e^{\frac{a}{2} u^{2}} d u \int_{0}^{u} v e^{\frac{a}{2} v^{2}} \phi(u, v) d v .
\end{aligned}
$$

But, some elementary calculus can show that

$$
\begin{aligned}
C_{H} a^{2} e^{-a T^{2}} s^{2+2 H}(t-s)^{2} & \leq A_{[0, s]^{2}} \leq 2 a^{2} s^{2+2 H}(t-s)^{2}, \\
e^{-\frac{a}{2}\left(t^{2}-s^{2}\right)}(t-s)^{2 H} & \leq A_{[s, t]^{2}} \leq(t-s)^{2 H}
\end{aligned}
$$

and

$$
\lim _{s \uparrow t} \frac{A_{[s, t] \times[0, s]}}{(t-s)^{2 H}}=0, \quad \lim _{s \downarrow 0} \frac{A_{[s, t] \times[0, s]}}{(t-s)^{2 H}}=0,
$$

which lead to

$$
\lim _{s \uparrow t} \frac{\sigma_{t, s}^{2}}{(t-s)^{2 H}}=1, \quad e^{-\frac{a}{2} t^{2}} t^{2 H} \leq \lim _{s \downarrow 0} \frac{\sigma_{t, s}^{2}}{(t-s)^{2 H}} \leq t^{2 H} .
$$

It follows that there are two constants $c_{a, H, T}, C_{a, H, T}>0$ such that

$$
c_{a, H, T}(t-s)^{2 H} \leq \sigma_{t, s}^{2} \leq C_{a, H, T}(t-s)^{2 H} .
$$

This completes the proof. 
From the lemma above, we see that

$$
\int_{0}^{t} \int_{0}^{t} E\left[\left(X_{u}^{H}-X_{v}^{H}\right)^{2}\right]^{-1 / 2} d u d v<\infty
$$

holds for all $t \geq 0$, and furthermore, we can show that the process $X^{H}=\left(X_{t}^{H}\right)_{0 \leq t \leq T}$ is local nondeterminism for every $0<T<\infty$, i.e. for $0 \leq t_{1}<t_{2}<\cdots<t_{n} \leq T$,

$$
\operatorname{Var}\left(\sum_{j=2}^{n} u_{j}\left(X_{t_{j}}^{H}-X_{t_{j-1}}^{H}\right)\right) \geq \kappa_{0} \sum_{j=2}^{n} u_{j}^{2} \sigma_{t_{j}, t_{j-1}}^{2}
$$

with a constant $\kappa_{0}>0$. Combining this with Berman [1, 2, we obtain

Proposition 4.2. If $\nu=0$, then the solution $X^{H}$ of the equation (1.3) has continuous local time $\mathscr{L}_{t}^{x}, t \geq 0, x \in \mathbb{R}$ such that

$$
\mathscr{L}_{t}^{x}=\lim _{\varepsilon \downarrow 0} \frac{1}{2 \varepsilon} \int_{0}^{t} 1_{[x-\varepsilon, x+\varepsilon]}\left(X_{s}^{H}\right) d s=\int_{0}^{t} \delta\left(X_{s}^{H}-x\right) d s,
$$

where $\delta\left(X_{s}^{H}-\cdot\right)$ denotes the delta function of $X_{s}^{H}$.

For $t \geq 0, x \in \mathbb{R}$ we now set

$$
\mathcal{L}_{t}^{x}=2 H(2 H-1) \int_{0}^{t} \delta\left(X_{s}^{H}-x\right) d s \int_{0}^{s} h(s, m)(s-m)^{2 H-2} d m .
$$

Then $\mathcal{L}_{t}^{x}$ is well-defined and

$$
\mathcal{L}_{t}^{x}=\int_{0}^{t} \delta\left(X_{s}^{H}-x\right) D_{s}^{\phi} X_{s}^{H} d s .
$$

The process $\left(\mathcal{L}_{t}^{x}\right)_{t \geq 0}$ is called the weighted local time of $X^{H}$ at $x \in \mathbb{R}$.

Lemma $4.3(\mathrm{Hu}[14])$. Let $Y$ be normally distributed with mean zero and variance $\sigma^{2}(\sigma>0)$. Then the delta function $\delta(Y-\cdot)$ of $Y$ exists uniquely and we have

$$
\delta(Y-x)=\frac{1}{2 \pi} \int_{\mathbb{R}} e^{i \xi(Y-x)} d \xi, \quad x \in \mathbb{R} .
$$

Proposition 4.4. Assume that $t \in[0, T]$ is given. Then $\mathcal{L}_{t}^{x}$ and $\mathscr{L}_{t}^{x}$ are square integrable for all $x \in \mathbb{R}$ and we have

$$
\begin{gathered}
E\left[\left(\mathscr{L}_{t}^{x}\right)^{2}\right] \leq C_{a, H, T} t^{2-2 H}, \\
E\left[\left(\mathcal{L}_{t}^{x}\right)^{2}\right] \leq C_{a, H, T} t^{2 H} .
\end{gathered}
$$

Proof. We have by Lemma 4.3

$$
\begin{aligned}
E\left[\left(\mathscr{L}_{t}^{x}\right)^{2}\right] & \leq \frac{1}{(2 \pi)^{2}} \int_{0}^{t} \int_{0}^{t} d u d v \int_{\mathbb{R}^{2}} E\left[e^{i\left(\xi X_{u}^{H}+\eta X_{v}^{H}\right)}\right] d \xi d \eta \\
& \leq \frac{2}{(2 \pi)^{2}} \int_{0}^{t} d u \int_{0}^{t} d v \int_{\mathbb{R}^{2}} e^{-\frac{1}{2} \operatorname{Var}\left(\xi X_{u}^{H}+\eta X_{v}^{H}\right)} d \xi d \eta .
\end{aligned}
$$

Noting that

$$
\operatorname{Var}\left(\xi X_{u}^{H}+\eta X_{v}^{H}\right) \geq k\left[\xi^{2} \sigma_{u, v}^{2}+(\eta+\xi)^{2} \sigma_{v}^{2}\right]
$$


for a positive $k>0$ by local nondeterminacy of the process $X^{H}$, we get

$$
\begin{aligned}
E\left[\left(\mathscr{L}_{t}^{x}\right)^{2}\right] & \leq \frac{2}{(2 \pi)^{2}} \int_{0}^{t} d u \int_{0}^{u} d v \int_{\mathbb{R}^{2}} e^{-\frac{k}{2}\left(\xi^{2} \sigma_{u, v}^{2}+(\eta+\xi)^{2} \sigma_{v}^{2}\right)} d \xi d \eta \\
& \leq \frac{1}{k \pi} \int_{0}^{t} d u \int_{0}^{u} \frac{d v}{\sigma_{u, v} \sigma_{v}} \\
& \leq \frac{1}{k \pi c_{a, T}} \int_{0}^{t} d u \int_{0}^{u} \frac{d v}{(u-v)^{H} v^{H}} \leq C_{a, H, T} T^{2-2 H}
\end{aligned}
$$

by Lemma 4.1. This obtains (4.6). Similarly, one can show that inequality (4.7) holds.

Theorem 4.5. Let $X^{H}$ be the solution to the equation (1.3) with Hurst index $\frac{1}{2}<$ $H<1, X_{0}^{H}=z, \nu=0$ and let $\mathcal{L}$ be the weighted local time of $X^{H}$. Suppose that $\Phi: \mathbb{R}^{+} \rightarrow \mathbb{R}$ is a convex function having polynomial growth. Then

$$
\Phi\left(X_{t}^{H}\right)=\Phi(z)+\int_{0}^{t} D^{-} \Phi\left(X_{s}^{H}\right) d X_{s}^{H}+\int_{\mathbb{R}} \mathcal{L}_{t}^{x} \mu_{\Phi}(d x),
$$

where $D^{-} \Phi$ denotes the left derivative of $\Phi$ and the signed measure $\mu_{\Phi}$ is defined by

$$
\mu_{\Phi}([a, b])=D^{-} \Phi(b)-D^{-} \Phi(a), \quad a<b, a, b \in \mathbb{R} .
$$

Proof. For $\varepsilon>0$ and $x \in \mathbb{R}$ we set

$$
\Phi_{\varepsilon}(x)=\int_{\mathbb{R}} p_{\varepsilon}(x-y) \Phi(y) d y \quad(\varepsilon>0),
$$

where $p_{\varepsilon}(x)=\frac{1}{\sqrt{2 \pi \varepsilon}} e^{-\frac{1}{2 \varepsilon} x^{2}}$. Then $\Phi_{\varepsilon} \in C^{2}$ and we have $\lim _{\varepsilon \downarrow 0} \Phi_{\varepsilon}(x)=\Phi(x)$, $\lim _{\varepsilon \downarrow 0} \Phi_{\varepsilon}^{\prime}(x)=D^{-} \Phi(x)$ for all $x \in \mathbb{R}$. It follows that for all $\varepsilon>0$

$$
\Phi_{\varepsilon}\left(X_{t}^{H}\right)=\Phi_{\varepsilon}(z)+\int_{0}^{t} \Phi_{\varepsilon}^{\prime}\left(X_{s}^{H}\right) d X_{s}^{H}+2 H(2 H-1) \int_{0}^{t} \Phi_{\varepsilon}^{\prime \prime}\left(X_{s}^{H}\right) \widetilde{h}(s) d s .
$$

On the other hand, it is easy to see that $\Phi_{\varepsilon}\left(X_{t}^{H}\right)$ converges to $\Phi\left(X_{t}^{H}\right)$ almost surely, and $\int_{0}^{t} \Phi_{\varepsilon}^{\prime}\left(X_{s}^{H}\right) X_{s}^{H} d s \rightarrow \int_{0}^{t} D^{-} \Phi\left(X_{s}^{H}\right) X_{s}^{H} d s$ a.s., and furthermore, $\int_{0}^{t} \Phi_{\varepsilon}^{\prime}\left(X_{s}^{H}\right) d B_{s}^{H} \rightarrow$ $\int_{0}^{t} D^{-} \Phi\left(X_{s}^{H}\right) d B_{s}^{H}$ in $(\mathcal{S})^{*}$.

Finally, we have as $\varepsilon \rightarrow 0$

$$
\begin{aligned}
\int_{0}^{t} \Phi_{\varepsilon}^{\prime \prime}\left(X_{s}^{H}\right) \widetilde{h}(s) d s & =\int_{0}^{t} d s \widetilde{h}(s) \int_{\mathbb{R}} \Phi_{\varepsilon}^{\prime \prime}(x) \delta\left(X_{s}^{H}-x\right) d x \\
& \rightarrow \frac{1}{2 H(2 H-1)} \int_{\mathbb{R}} \mathcal{L}_{t}^{x} \mu_{\Phi}(d x) .
\end{aligned}
$$

This completes the proof.

Corollary 4.6. Let $X^{H}$ be the solution to the equation (1.3) with Hurst index $\frac{1}{2}<$ $H<1, X_{0}^{H}=z, \nu=0$ and let $\mathcal{L}$ be the weighted local time of $X^{H}$. Then the Tanaka formula

$$
\left|X_{t}^{H}-x\right|=\left|X_{0}^{H}-x\right|+\int_{0}^{t} \operatorname{sign}\left(X_{s}^{H}-x\right) d X_{s}^{H}+\mathcal{L}_{t}^{x}
$$

holds for all $x \in \mathbb{R}$. 


\section{Self-intersection local time on $\mathbb{R}^{2}$}

In this section, we shall use the idea of $\mathrm{Hu}$ [12] (see also Hu-Nualart [13]) to study the the renormalized self-intersection local time of the linear fractional selfattracting diffusion $X^{H}=\left(X^{H, 1}, X^{H, 2}\right)$ on $\mathbb{R}^{2}$, where $X^{H, j}(j=1,2)$ is the solution of the equation

$$
X_{t}^{H, j}=B_{t}^{H, j}-a \int_{0}^{t} \int_{0}^{u}\left(X_{u}^{H, j}-X_{v}^{H, j}\right) d v d u, \quad 0 \leq t \leq T
$$

with $a>0$ and two independent fractional Brownian motions $B_{t}^{H, j}, j=1,2$. Then we have

$$
X_{t}^{H, j}=\int_{0}^{t} h(t, s) d B_{t}^{H, j}, \quad j=1,2
$$

from Section 3, and for all $s, t \geq 0$

$$
h(t, s)= \begin{cases}1-a s e^{\frac{1}{2} a s^{2}} \int_{s}^{t} e^{-\frac{1}{2} a u^{2}} d u, & t \geq s \\ 0, & t<s .\end{cases}
$$

The renormalized self-intersection local time $\beta_{T}^{H}$ of the process

$$
X_{t}^{H}=\left(X_{t}^{H, 1}, X_{t}^{H, 2}\right), \quad 0 \leq t \leq T
$$

is formally defined as

$$
\beta_{T}^{H}=\int_{0}^{T} \int_{0}^{t} \delta_{0}\left(X_{t}^{H}-X_{s}^{H}\right) d s d t-E\left[\int_{0}^{T} \int_{0}^{t} \delta_{0}\left(X_{t}^{H}-X_{s}^{H}\right) d s d t\right],
$$

where $\delta_{0}$ is the delta function. For $\varepsilon>0$ we define

$$
\beta_{T}^{H, \varepsilon}=\int_{0}^{T} \int_{0}^{t} p_{\varepsilon}\left(X_{t}^{H}-X_{s}^{H}\right) d s d t
$$

where

$$
p_{\varepsilon}(x)=\frac{1}{2 \pi \varepsilon} e^{-\frac{|x|^{2}}{2 \varepsilon}}, \quad x \in \mathbb{R}^{2}
$$

is the heat kernel. Then main object of this section is to explain and prove Theorem 5.1 .

Theorem 5.1. The random variable $\beta_{T}^{H, \varepsilon}-E\left[\beta_{T}^{H, \varepsilon}\right]$ converges in $L^{2}$ as $\varepsilon$ tends to zero if $\frac{1}{2}<H<\frac{3}{4}$.

In order to prove the theorem we need some preliminaries. For $t \geq s \geq 0, t^{\prime} \geq$ $s^{\prime} \geq 0$ we now denote

$$
\sigma_{t, s}^{2}=E\left(X_{t}^{H, 1}-X_{s}^{H, 1}\right)^{2}, \quad \mu=E\left(X_{t}^{H, 1}-X_{s}^{H, 1}\right)\left(X_{t^{\prime}}^{H, 1}-X_{s^{\prime}}^{H, 1}\right)
$$

and

$$
d_{H}\left(s, t, s^{\prime}, t^{\prime}\right)=\sigma_{s, t^{\prime}}^{2} \sigma_{s^{\prime}, t^{\prime}}^{2}-\mu^{2} .
$$

Then, by Lemma 4.1 and $\mathrm{Hu}$ [12] one can establish the following lemma. 
Lemma 5.2. (1) For $0<s<s^{\prime}<t<t^{\prime}<T$, we have

$$
d_{H}\left(s, t, s^{\prime}, t^{\prime}\right) \geq \kappa\left[(t-s)^{2 H}\left(t^{\prime}-t\right)^{2 H}+\left(t^{\prime}-s^{\prime}\right)^{2 H}\left(s^{\prime}-s\right)^{2 H}\right] .
$$

(2) For $0<s^{\prime}<s<t<t^{\prime}<T$, we have

$$
d_{H}\left(s, t, s^{\prime}, t^{\prime}\right) \geq \kappa(t-s)^{2 H}\left(t^{\prime}-s^{\prime}\right)^{2 H} .
$$

(3) For $0<s<t<s^{\prime}<t^{\prime}<T$, we have

$$
d_{H}\left(s, t, s^{\prime}, t^{\prime}\right) \geq \kappa(t-s)^{2 H}\left(t^{\prime}-s^{\prime}\right)^{2 H},
$$

where $\kappa>0$ is an enough small constant.

Lemma 5.3. For $0 \leq x<y \leq T$ we set

$$
\begin{aligned}
& h^{*}(y, x, u, v) \\
& \quad=\left[h(y, u) 1_{(0, y]}(u)-h(x, u) 1_{(0, x]}(u)\right]\left[h(y, v) 1_{(0, y]}(v)-h(x, v) 1_{(0, x]}(v)\right],
\end{aligned}
$$

where $h(\cdot, \cdot)$ is defined in Section 3. Then we have

$$
\begin{aligned}
& \int_{0}^{t^{\prime}} \int_{0}^{t^{\prime}}\left[h^{*}\left(t^{\prime}, s, u, v\right)-\right.\left.h^{*}\left(t^{\prime}, t, u, v\right)\right] \phi(u, v) d u d v \\
& \leq C_{a, H, T}\left[\left(t^{\prime}-s\right)^{2 H}-\left(t^{\prime}-t\right)^{2 H}\right]
\end{aligned}
$$

for all $0 \leq s \leq t \leq t^{\prime} \leq T$.

Proof. For $0<u, v<T$ we have

$$
h\left(t^{\prime}, u\right) 1_{\left(0, t^{\prime}\right]}(u)-h(s, u) 1_{(0, s]}(u)=h\left(t^{\prime}, u\right) 1_{\left(s, t^{\prime}\right]}(u)+\left[h\left(t^{\prime}, u\right)-h(s, u)\right] 1_{(0, s]}(u),
$$

and

$$
h\left(t^{\prime}, v\right) 1_{\left(0, t^{\prime}\right]}(v)-h(s, v) 1_{(0, s]}(v)=h\left(t^{\prime}, v\right) 1_{\left(s, t^{\prime}\right]}(v)+\left[h\left(t^{\prime}, v\right)-h(s, v)\right] 1_{(0, s]}(v) .
$$

So,

$$
\begin{aligned}
h^{*}\left(t^{\prime}, s, u, v\right) & =h\left(t^{\prime}, u\right) h\left(t^{\prime}, v\right) 1_{\left(s, t^{\prime}\right]^{2}}(u, v) \\
& +\left[h\left(t^{\prime}, u\right)-h(s, u)\right]\left[h\left(t^{\prime}, v\right)-h(s, v)\right] 1_{(0, s]^{2}}(u, v) \\
& -h\left(t^{\prime}, u\right)\left[h(s, v)-h\left(t^{\prime}, v\right)\right] 1_{\left(s, t^{\prime}\right]}(u) 1_{(0, s]}(v) \\
& -h\left(t^{\prime}, v\right)\left[h(s, u)-h\left(t^{\prime}, u\right)\right] 1_{(0, s]}(u) 1_{\left(s, t^{\prime}\right]}(v) .
\end{aligned}
$$

Similarly, we also have

$$
\begin{aligned}
h^{*}\left(t^{\prime}, t, u, v\right) & =h\left(t^{\prime}, u\right) h\left(t^{\prime}, v\right) 1_{\left(t, t^{\prime}\right]^{2}}(u, v) \\
& +\left[h\left(t^{\prime}, u\right)-h(t, u)\right]\left[h\left(t^{\prime}, v\right)-h(t, v)\right] 1_{(0, t]^{2}}(u, v) \\
& -h\left(t^{\prime}, u\right)\left[h(t, v)-h\left(t^{\prime}, v\right)\right] 1_{\left(t, t^{\prime}\right]}(u) 1_{(0, t]}(v) \\
& -h\left(t^{\prime}, v\right)\left[h(t, u)-h\left(t^{\prime}, u\right)\right] 1_{(0, t]}(u) 1_{\left(t, t^{\prime}\right]}(v) .
\end{aligned}
$$


On the other hand, for all $0<u, v \leq s \leq t \leq t^{\prime} \leq T$ we set

$$
\begin{aligned}
\Delta\left(t^{\prime}, t, s, u, v\right) \equiv[h(s, u) & \left.-h\left(t^{\prime}, u\right)\right]\left[h(s, v)-h\left(t^{\prime}, v\right)\right] 1_{(0, s]^{2}}(u, v) \\
& -\left[h(t, u)-h\left(t^{\prime}, u\right)\right]\left[h(t, v)-h\left(t^{\prime}, v\right)\right] 1_{(0, t]^{2}}(u, v) \\
= & a^{2} u v e^{\frac{a}{2}\left(u^{2}-v^{2}\right)}\left\{\left(\int_{s}^{t^{\prime}} e^{-\frac{a}{2} w^{2}} d w\right)^{2}-\left(\int_{t}^{t^{\prime}} e^{-\frac{a}{2} w^{2}} d w\right)^{2}\right\} .
\end{aligned}
$$

Then for all $s<t \leq t^{\prime} \leq T$, we have

$$
\lim _{s \downarrow 0} \frac{1}{\left(t^{\prime}-s\right)^{2 H}-\left(t^{\prime}-t\right)^{2 H}} \int_{0}^{s} \int_{0}^{s} \Delta\left(t^{\prime}, t, s, u, v\right) \phi(u, v) d u d v=0
$$

and

$$
\lim _{s \uparrow t} \frac{1}{\left(t^{\prime}-s\right)^{2 H}-\left(t^{\prime}-t\right)^{2 H}} \int_{0}^{s} \int_{0}^{s} \Delta\left(t^{\prime}, t, s, u, v\right) \phi(u, v) d u d v=0,
$$

which implies that there is a constant $C_{a, H, T}>0$ such that

$$
\int_{0}^{s} \int_{0}^{s} \Delta\left(t^{\prime}, t, s, u, v\right) \phi(u, v) d u d v \leq C_{a, H, T}\left[\left(t^{\prime}-s\right)^{2 H}-\left(t^{\prime}-t\right)^{2 H}\right] .
$$

Combining these with

$$
0 \leq h(t, u)-h\left(t^{\prime}, u\right) \leq h(s, u)-h\left(t^{\prime}, u\right) \leq 2, \quad 0 \leq u \leq s \leq t,
$$

we get

$$
\begin{gathered}
\int_{0}^{t^{\prime}} \int_{0}^{t^{\prime}}\left[h^{*}\left(t^{\prime}, s, u, v\right)-h^{*}\left(t^{\prime}, t, u, v\right)\right] \phi(u, v) d u d v \\
\leq \int_{0}^{t^{\prime}} \int_{0}^{t^{\prime}} h\left(t^{\prime}, u\right) h\left(t^{\prime}, v\right)\left(1_{\left(s, t^{\prime}\right]^{2}}(u, v)-1_{\left(t, t^{\prime}\right]^{2}}(u, v)\right) \phi(u, v) d u d v \\
\quad+4 \int_{t}^{t^{\prime}} \int_{s}^{t} \phi(u, v) d u d v \\
\quad+\int_{0}^{s} \int_{0}^{s} \Delta\left(t^{\prime}, t, s, u, v\right) \phi(u, v) d u d v \\
\leq C_{a, H, T}\left[\left(t^{\prime}-s\right)^{2 H}-\left(t^{\prime}-t\right)^{2 H}\right] .
\end{gathered}
$$

This completes the proof.

The proof similar to Lemma 5.3, by decomposing the function

$$
h^{*}\left(t^{\prime}, s, u, v\right)-h^{*}\left(t^{\prime}, t, u, v\right)+h^{*}\left(s^{\prime}, t, u, v\right)-h^{*}\left(s^{\prime}, s, u, v\right),
$$

one can show that the following lemma holds for all $0 \leq s \leq t \leq s^{\prime} \leq t^{\prime} \leq T$.

Lemma 5.4. Under the assumptions of Lemma 5.3, for all $0 \leq s \leq t \leq s^{\prime} \leq t^{\prime} \leq T$ we have

$$
\begin{gathered}
\int_{0}^{t^{\prime}} \int_{0}^{t^{\prime}}\left[h^{*}\left(t^{\prime}, s, u, v\right)-h^{*}\left(t^{\prime}, t, u, v\right)+h^{*}\left(s^{\prime}, t, u, v\right)-h^{*}\left(s^{\prime}, s, u, v\right)\right] \phi(u, v) d u d v \\
\leq C_{a, H, T}\left[\left(t^{\prime}-s\right)^{2 H}-\left(t^{\prime}-t\right)^{2 H}+\left(s^{\prime}-t\right)^{2 H}-\left(s^{\prime}-s\right)^{2 H}\right] .
\end{gathered}
$$


Lemma 5.5. Let $\sigma_{t, s}^{2}$ and $\mu$ be as in the proof of Theorem 5.1. Then we have

$$
\int_{\mathbb{T}} \frac{\mu^{2} d s d t d s^{\prime} d t^{\prime}}{d_{H}\left(s, t, s^{\prime}, t^{\prime}\right)\left(\sigma_{t, s}^{2} \sigma_{t^{\prime}, s^{\prime}}^{2}\right)}<\infty
$$

if $\frac{1}{2}<H<\frac{3}{4}$.

Lemma 5.5 is a consequence of the lemmas above and Lemma 15 in $\mathrm{Hu}$ [13] (see also $\mathrm{Hu}$ [12, pp.245-247]). In fact, Lemma 5.3 and Lemma 5.4 imply that the estimate

$$
\mu \leq C_{a, H, T}\left[\left|t-s^{\prime}\right|^{2 H}-\left|t^{\prime}-t\right|^{2 H}+\left|t^{\prime}-s\right|^{2 H}-\left|s-s^{\prime}\right|^{2 H}\right]
$$

holds for all $\left(s, s^{\prime}, t, t^{\prime}\right) \in \mathbb{T}$. Thus, Lemma 5.5 follows from Lemma 5.2 and Lemma 15 in $\mathrm{Hu}$ [13] (see also $\mathrm{Hu}$ [12, pp.245-247]).

Now we can prove Theorem 5.1 .

Proof of Theorem 5.1. Clearly, as $\varepsilon$ tends to zero $\beta_{T}^{H, \varepsilon}-E\left[\beta_{T}^{H, \varepsilon}\right]$ converges in $L^{2}$ if and only if

$$
\operatorname{Var}\left(\beta_{T}^{H, \varepsilon}\right)=E\left[\left(\beta_{T}^{H, \varepsilon}\right)^{2}\right]-\left(E\left(\beta_{T}^{H, \varepsilon}\right)\right)^{2}
$$

tends to a constant. Now let us show that $\operatorname{Var}\left(\beta_{T}^{H, \varepsilon}\right)$ converges as $\varepsilon$ tends to zero. Using the classical equality

$$
p_{\varepsilon}(x)=\frac{1}{(2 \pi)^{2}} \int_{\mathbb{R}^{2}} e^{i<\xi, x>} e^{-\varepsilon \frac{|\xi|^{2}}{2}} d \xi
$$

one can obtain

$$
\beta_{T}^{H, \varepsilon}=\frac{1}{(2 \pi)^{2}} \int_{0}^{T} \int_{0}^{t} \int_{\mathbb{R}^{2}} e^{i<\xi, X_{t}^{H}-X_{s}^{H}>} e^{-\varepsilon \frac{|\xi|^{2}}{2}} d \xi d s d t .
$$

Combining this with the facts $<\xi, X_{t}^{H}-X_{s}^{H}>\sim N\left(0,|\xi|^{2} \sigma_{t, s}^{2}\right)$ and

$$
\begin{gathered}
E\left[e^{i<\xi, X_{t}^{H}-X_{s}^{H}>}\right]=e^{-\frac{1}{2}|\xi|^{2} \sigma_{t, s}^{2}}, \\
\int_{\mathbb{R}^{2}} e^{-\frac{1}{2}|\xi|^{2}\left(\varepsilon+\sigma^{2}(t, s)\right)} d \xi=\frac{2 \pi}{\varepsilon+\sigma_{t, s}^{2}},
\end{gathered}
$$

we get

$$
\begin{aligned}
E\left[\beta_{T}^{H, \varepsilon}\right] & =\int_{0}^{T} \int_{0}^{t} E\left(p_{\varepsilon}\left(X_{t}^{H}-X_{s}^{H}\right)\right) d s d t \\
& =\frac{1}{2 \pi} \int_{0}^{T} \int_{0}^{t}\left(\varepsilon+\sigma_{t, s}^{2}\right)^{-1} d s d t .
\end{aligned}
$$

Denote $\mathbb{T}=\left\{\left(s, t, s^{\prime}, t^{\prime}\right): 0<s<t<T, 0<s^{\prime}<t^{\prime}<T\right\}$, then according to the representation (5.5) we get

$$
E\left[\left(\beta_{T}^{H, \varepsilon}\right)^{2}\right]=\frac{1}{(2 \pi)^{4}} \int_{\mathbb{T}} \int_{\mathbb{R}^{4}} E e^{i<\xi, X_{t}^{H}-X_{s}^{H}>+i<\eta, X_{t^{\prime}}^{H}-X_{s^{\prime}}^{H}>} e^{-\varepsilon \frac{|\xi|^{2}+|\eta|^{2}}{2}} d \xi d \eta d s d t d s^{\prime} d t^{\prime} .
$$

Noting that

$$
<\xi, X_{t}^{H}-X_{s}^{H}>+<\eta, X_{t^{\prime}}^{H}-X_{s^{\prime}}^{H}>\sim N\left(0,|\xi|^{2} \sigma_{t, s}^{2}+|\eta|^{2} \sigma_{t^{\prime}, s^{\prime}}^{2}+2 \mu<\xi, \eta>\right)
$$


for any $\xi, \eta \in \mathbb{R}^{2}$, we can write

$$
\begin{aligned}
E\left[\left(\beta_{T}^{H, \varepsilon}\right)^{2}\right] & =\frac{1}{(2 \pi)^{4}} \int_{\mathbb{T}} \int_{\mathbb{R}^{4}} e^{-\frac{1}{2}\left(\left(\sigma_{t, s}^{2}+\varepsilon\right)|\xi|^{2}+2 \mu<\xi, \eta>+\left(\sigma_{t^{\prime}, s^{\prime}}^{2}+\varepsilon\right)|\eta|^{2}\right)} d \xi d \eta d s d t d s^{\prime} d t^{\prime} \\
& =\frac{1}{(2 \pi)^{2}} \int_{\mathbb{T}}\left(\left(\sigma_{t, s}^{2}+\varepsilon\right)\left(\sigma_{t^{\prime}, s^{\prime}}^{2}+\varepsilon\right)-\mu^{2}\right)^{-d / 2} d s d t d s^{\prime} d t^{\prime}
\end{aligned}
$$

for all $\varepsilon>0$. It follows from (5.6) that

$$
\begin{aligned}
E\left[\left(\beta_{T}^{H, \varepsilon}\right)^{2}\right]- & \left(E \beta_{T}^{H, \varepsilon}\right)^{2}=\frac{1}{(2 \pi)^{2}} \int_{\mathbb{T}}\left[\left(\left(\sigma_{t, s}^{2}+\varepsilon\right)\left(\sigma_{t^{\prime}, s^{\prime}}^{2}+\varepsilon\right)-\mu^{2}\right)^{-1}-\right. \\
& =\frac{1}{(2 \pi)^{2}} \int_{\mathbb{T}} \frac{\left.\left(\left(\varepsilon+\sigma_{t, s}^{2}\right)\left(\varepsilon+\sigma_{t^{\prime}, s^{\prime}}^{2}\right)\right)^{-1}\right] d s d t d s^{\prime} d t^{\prime}}{\left(\left(\sigma_{t, s}^{2}+\varepsilon\right)\left(\sigma_{t^{\prime}, s^{\prime}}^{2}+\varepsilon\right)-\mu^{2}\right)\left(\varepsilon+\sigma_{t, s}^{2}\right)\left(\varepsilon+\sigma_{t^{\prime}, s^{\prime}}^{2}\right)} .
\end{aligned}
$$

Thus, the theorem follows from Lemma 5.5 .

\section{ACKNOWLEDGEMENT}

The authors would like to thank the anonymous referees for the careful reading of the manuscript and many helpful comments.

\section{REFERENCES}

[1] Berman, S.M. (1969). Local times and sample function properties of stationary Gaussian processes. Trans. Amer. Math. Soc. 137, 277-299.

[2] Berman, S.M. (1973). Local nondeterminism and local times of Gaussian processes. Indiana Univ. Math. J. 23, 69-74.

[3] Chakravarti N. and Sebastian K.L. (1997). Fractional Brownian Motion Model for polymers. Chemical Physics Letters 267, 9-13.

[4] Cherayil J. and Biswas P. (1993). Path integral description of polymers using fractional Brownian walks. The Journal of Chemical Physics 11, 9230-9236.

[5] Cranston M. and Le Jan Y. (1995). Self-attracting diffusions: two case studies. Math. Ann. 303, 87-93.

[6] Duncan T.E., Hu Y. and Duncan B.P. (2000). Stochastic calculus for fractional Brownian motion, I Theory. SIAM J. Control Optim. 38, 582-612.

[7] Durrett R. and Rogers L.C.G. (1991). Asymptotic behavior of Brownian polymer. Prob. Theory Rel. Fields 92, 337-349.

[8] Elliott R.J. and Van der Hoek J. (2003). A general fractional white noise theory and applications to finance. Math. Finance 13, 301-330.

[9] Herrmann S. and Roynette B. (2003). Boundedness and convergence of some self-attracting diffusions. Math. Ann. 325, 81-96.

[10] Herrmanna S. and Scheutzow M. (2004). Rate of convergence of some self-attracting diffusions. Stoc. Proc. Appl. 111, 41-55.

[11] Hu Y. (2005). Integral Transformations and Anticipative Calculus for Fractional Brownian Motions. Memoirs Amer. Math. Soc. Vol. 175, No. 825.

[12] Hu Y. Self-intersection local time for fractional Brownian motions-via chaos expansion. $J$. Math. Kyoto Univ. 41 (2001), 233-250.

[13] $\mathrm{Hu}$ Y. and Nualart D. (2005). Renormalized self-intersection local time for fractional Brownian motion. Ann. Prob. 33, 948-983. 
[14] $\mathrm{Hu}$ Y. and Øksendal B. (2002). Chaos expansion of local time of fractional Brownian motions. Stochastic Anal. Appl. 20, 815-837.

[15] $\mathrm{Hu}$ Y. and Øksendal B. (2003). Fractional white nosie calculus and applications to finance. Infinite Dimensional Analysis, Quantum Probability and Related Topics 6, 1-32.

[16] Mandelbrot B.B. and Van Ness J.W. (1968). Fractional Brownian motion, fractional noises and applications. SIAM Review 10, 422-437.

[17] Nualart D. (2003) Stochastic integration with respect to fractional Brownian motion and applications. Contemporary Mathematics 336, 3-39.

[18] Nualart D. (2006) Malliavin Calculus and Related Topics, 2nd edn. Springer-Verlag.

[19] Øksendal B. (2003). Fractional Brownian motion in finance. Preprint-series in Oslo University, Pure Mathematics 13, 1-39.

[20] Pemantle R. (1988). Phase transition in reinforced random walk and RWRE on trees. Ann. Probab. 16, 1229-1241.

[21] Sebastian K. L. (1995). Path integral representation for fractional Brownian motion. J. Phys. A 28, 4305-4311. 